EOMmun Communication et organisation

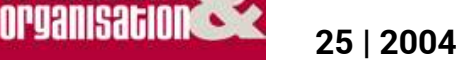

Les vallées : sens, territoires et signes

\title{
La médiation de la biodiversité
}

\section{Francine Boillot-Gren}

\section{OpenEdition}

Journals

Édition électronique

URL : http://journals.openedition.org/communicationorganisation/2964

DOI : 10.4000/communicationorganisation.2964

ISSN : 1775-3546

\section{Éditeur}

Presses universitaires de Bordeaux

\section{Édition imprimée}

Date de publication : 2 octobre 2004

ISSN : 1168-5549

\section{Référence électronique}

Francine Boillot-Gren, « La médiation de la biodiversité », Communication et organisation [En ligne],

25 | 2004, mis en ligne le 27 mars 2012, consulté le 01 mai 2019. URL : http://

journals.openedition.org/communicationorganisation/2964; DOI : 10.4000/

communicationorganisation.2964

Ce document a été généré automatiquement le 1 mai 2019.

(c) Presses universitaires de Bordeaux 


\title{
La médiation de la biodiversité
}

\author{
Francine Boillot-Gren
}

\section{Introduction}

1 En 1999, le Parc National du Mercantour cherche à élaborer un appel d'offre concernant une exposition sur la biodiversité destinée à la future maison du Parc à Tende ${ }^{1}$. Ceci me donne l'occasion de m'interroger sur les indicateurs susceptibles d'encadrer l'élaboration d'un projet de médiation sur la biodiversité. Ce travail s'inscrit dans une recherche muséologique plus vaste menée sur les Parcs nationaux. A l'instar de l'ICOM, je considère les «territoires - Parcs nationaux » comme des entités muséologiques à part entière. En effet, ils ont pour mission la conservation, la recherche, l'accueil du public (éducationloisirs) et l'aide au développement durable. Des missions qui rencontrent parfaitement la définition de la muséologie ouverte définie par G.H. Rivière (1989) ${ }^{2}$. Le projet de médiation du Parc national témoigne également de l'ouverture actuelle des musées vers la société qui tentent de prendre en compte une grande diversité de publics, de mettre en place des stratégies communicationnelles multiples, intra et extra-muros, fédérant plusieurs thématiques, approches, sensibilités et de fonctionner au sein d'un vaste système partenariat socioéconomique, politique, scientifique et culturel. (J. Davallon, G. Grandmont, B. Schiele, 1992). Je ne peux donc imaginer désolidariser cette exposition et sa thématique de son territoire. Par ailleurs, j'entends ici la médiation comme une mise en relation (au sens souvent attribué à la communication, par exemple, par D. Bougnoux, 1998) mais, aussi comme une transmission d'un héritage culturel et naturel en pleine transformation ( $R$. Debray, 1997). Appliquée à un espace habité et protégé, la médiation est donc ici une médiation naturaliste et culturelle qui vise à être une mise en liaison ou en reliaison destinée à résoudre les crises ${ }^{3}$ et à favoriser de nouvelles alliances sociales et territoriales (M. de Certeau, 1993). 


\section{Du contexte de la médiation à son concept}

2 Mes travaux dans les Parcs nationaux visent à dégager un cadre de référence pour la mise en place de la politique générale de la médiation des «territoires Parc national ${ }^{4}$ ». Ils relèvent combien un projet de médiation notamment scientifique est avant tout un processus de communication, siège de tensions et d'enjeux multiples, qui impose une clarification contextuelle approfondie. L'exposition sur la biodiversité dépend ainsi d'un triple contexte : celui d'une institution étatique et scientifique (le Parc national), celui d'une communauté locale franco-italienne (la vallée de La Roya-Bevera) et celui de l'espace lui-même (un élément spatiotemporel de la biosphère protégé, habité, traversé, projeté). Il apparaît nécessaire de fonder la définition de son projet muséal sur une approche équilibrée des réalités et des représentations et des finalités inhérentes à chaque acteur du territoire. L'ouverture du pro]et de médiation dépend ainsi de la clarification contextuelle puis de la négociation engagée entre les différents sens donnés au territoire qui inclut l'exposition (F. Boillot, 1998, 2002). ${ }^{5}$

Cela dit, l'exposition sur la biodiversité de Tende m'intéresse surtout parce qu'elle est proche de la programmation. Elle me permet ainsi d'approfondir un autre type de clarification : la clarification conceptuelle qui a l'avantage de concerner particulièrement la part « transmission » » de la médiation.

4 Notre société est aujourd'hui marquée par un désir mutuel de reconnaissance à des fins notamment politiques, médiatiques et économiques. La promotion du culturel (pluralité du lien) se fait au détriment de l'objet de culture (transcendance) (D. Bougnoux, 1998). Le contenu de la médiation culturelle a ainsi tendance à perdre de sa substance. Les identités locales disparaissent ou se banalisent comme les propositions muséales. Dans ces conditions, il importe de revaloriser l'ambition et la clarification conceptuelle du contenu de la médiation. Elle doit pleinement retrouver sa fonction de transmission au sens d'un échange de signes approfondi et vertical car participant au transfert et à l'évolution d'un héritage naturaliste et culturel (R. Debray, 1993). Or une institution étatique et scientifique comme un Parc national a à louer un rôle prépondérant dans le projet de transmission. La médiologie souligne combien la présence d'un cadre pérenne et structurant est nécessaire à la réussite d'une transmission ambitieuse obligatoirement lente et redondante. Cette institution a l'avantage également d'être du côté de la production de connaissances propres à nourrir le contenu de la transmission. Mais cette proximité avec le monde scientifique porte aussi en elle les germes d'une clôture sciences - société largement dénoncée.

5 L'exposition sur la biodiversité est donc un moment intéressant à interroger. Elle me donne l'opportunité de cerner les contours d'une médiation scientifique idéale et, notamment, de solliciter ses capacités à quitter un modèle ancien le plus souvent marqué du côté des émetteurs scientifiques, par une transmission élitiste, positiviste, dominatrice et du côté des émetteurs socioéconomiques, par une communication culturelle superficielle, stéréotypée, universalisante et banalisante (F. Boillot, 2004). Ce moment proche de la programmation m'amène surtout à interroger la clarification conceptuelle du contenu de l'exposition et par là, celle du thème très contemporain de la biodiversité. 


\section{La clarification conceptuelle de la thématique « biodiversité »}

6 Une exposition sur la biodiversité doit aboutir à une représentation actualisée et complète de la thématique et fournir une démonstration qui serve au mieux les finalités, l'identité et les publics du projet scientifique et culturel muséal (P. Rasse, 1997). La clarification conceptuelle (ou la définition) de la thématique «biodiversité » est donc un préalable essentiel à la définition du projet muséal. La grille conceptuelle attendue, trop vaste pour être communiquée dans son entier, constitue un outil d'aide à la décision lors de la phase de sélection.

Cependant bâtir une telle grille ne consiste pas seulement à recueillir, qualifier et organiser les connaissances. Une grille conceptuelle, aussi élargie soit-elle, n'est ni exhaustive ni neutre. La définition de la biodiversité ne peut être que le résultat d'une négociation permanente. Je m'appuie ici sur une précédente réflexion sur l'environnement, une notion proche et aussi englobante que celle de la biodiversité ( $\mathrm{F}$. Boillot, 1999). Je citerai, entre autres, J. Theys (1993) qui parle d'une définition de l'environnement impossible car trop polysémique et qui situe la définition au centre d'une négociation permanente sur les frontières entre espace publie et espace privé, culture el nature, logique technique et logique du vivant, mondes des systèmes et monde vécu. Ou encore, $\mathrm{L}$. Sauvé (1994) qui pose cette définition comme obligatoirement dépendante d'une réalité contextuelle donnée.

8 Je ne commencerai donc pas par lister les concepts de la biodiversité, mais poserai d'abord la définition de la biodiversité comme le résultat d'une négociation permanente entre plusieurs interprétations. Je relèverai au départ les conditions de l'interprétation et la pluralité des interprétations potentielles. Étant donnée la place de la science dans ce projet, je soulignerai le codage disciplinaire de cette interprétation et son impact sur l'efficacité de la médiation. De même, l'insertion de la médiation dans un projet de territoire me conduira à situer la transformation répétée de ces interprétations et ainsi à définir non pas les termes de la biodiversité mais sa dynamique. Puis, je tenterai un repérage conceptuel en proposant des indicateurs liés aux valeurs associées aujourd'hui à la biodiversité. Enfin, je situerai tout au long de la démonstration puis dans le dernier sous-chapitre, les éléments propres à animer un débat constructif de façon à valoriser le rôle émergent d'animation sociale du musée, lorsqu'il veut idéalement apparaître comme un musée d'interaction sociale ou d'implication' ${ }^{6}$ (J. Davallon et Al., 1992).

\section{La biodiversité, une dynamique en constante négociation}

\section{La biodiversité, une interprétation du réel parmi d'autres}

Le lieu ne donne pas un paysage mais une infinité d'histoires du monde en constant développement (F. Béguin, 1998). Ce point de vue très fréquent chez les philosophes - esthéticiens (par exemple, F. Dagognet, 1982), ou encore chez certains géographes et paysagistes (par exemple, A. Berque, 1990 ou M. Corajoud, 1982) s'appuie sur un point simple et fondamental de la sémiologie. Dès notre premier regard, l'interprétation agit. Comme le 
souligne C. Pierce (1978), la sémiosphère double et englobe la biosphère. La pluralité des points de vue (des interprétants, au sens de C. Pierce) conduit à une pluralité d'opérations paysagères.

Si l'on envisage l'exposition sur la biodiversité de Tende comme un projet de médiation et de territoire, elle doit idéalement mettre en visibilité et en débat l'ensemble de ces regards, l'ensemble de ces traductions du réel.

\section{La biodiversité pluridisciplinaire et pluridimensionnelle}

11 La biodiversité s'entend le plus communément comme les éléments génétiques, les espèces et les populations, les écosystèmes et les processus naturels qui assurent la perpétuation de la vie sous toutes ses formes (OCDE, Etat de l'environnement, 1991). Cette définition est très liée à un discours écologue. Malgré le souci d'objectivité propre à toute discipline scientifique, l'écologie a, comme les autres, une façon particulière de voir, sélectionner et catégoriser les objets de la biosphère. Si l'on considère à l'instar de J. Theys (1993) qu'une vision idéale de l'environnement devrait comporter cinq dimensions ou systèmes de sens, les écologues ont en général tendance à privilégier, comme ici, la dimension écologique (la faune, la flore, l'habitat) et la dimension sécuritaire (les problèmes d'environnement, la nature menacée). Par contre, ils négligent souvent les dimensions sociale, économique, culturelle et esthétique. La représentation du paysage ou de la biosphère risque donc d'être partielle si elle se cantonne à une lecture naturaliste ou bien sûr, à toute autre lecture disciplinaire.

12 Je retiendrai l'importance de donner une interprétation pluridisciplinaire et pluridimensionnelle de la biodiversité qui intègre aussi bien l'écosystème que le sociosystème, la nature que la culture ou autrement dit, les cinq dimensions précitées par J. Theys. ${ }^{7}$

\section{La biodiversité, un réel codé par le scientifique et l'habitant et ressenti par le visiteur}

Le matériau d'une exposition sur la biodiversité est issu généralement de nombreuses recherches. Même si on veille à leur pluridisciplinarité, il importe aussi de rappeler que la biosphère y sera nommée selon des codes disciplinaires qui en donnent une représentation abstraite, plus proche du logos que de l'indiciel (au sens du triangle sémiotique de C. Pierce, 1978), c'est-à-dire très éloignée de la chose commune, de ce réel que nous ne connaitrons jamais vraiment. Ce double travail, -interprétation et abstraction -, est à l'oeuvre dans toute lecture disciplinaire, celle des sciences naturelles comme celle des sciences humaines. Il est aussi présent dans la lecture communautaire circonscrite dans une culture qui, par définition, est aussi un construit codé. Ces lectures nourrissent le projet de transmission, mais font terriblement obstacle au pro)et conjoint de communication.

Interroger la biodiversité revient donc à poser clairement aussi la question de l'orientation el de l'abstraction de l'interprétation de toute discipline et de toute culture puis celle de sa difficile médiation.

En effet, cette sorte d'écran scientifique et culturel posé entre le monde et le récepteur ne rend pas compte de ce qui, du territoire, est le plus facilement perceptible par un visiteur néophyte et étranger à l'espace. Car, le lieu exprime d'abord une ambiance indicielle au 
sens de C. Peirce, c'est-à-dire il met plus facilement à disposition une série de signes peu déformés, peu codés, proches de la chose réelle. Les muséologues, les architectes, les paysagistes appellent cela la scénographie naturelle des lieux ou encore l'esprit du lieu (A. Viel, 1994). Ce sont ces éléments qui sont opérationnels du point de vue où je me place, c'est-à-dire celui de la communication et de la mise en relation. La recherche de la scénographie naturelle des lieux appelle des méthodes d'investigation particulières où l'espace est parcouru, vécu, ressenti, comparé selon des trajets et des démarches sensibles subjectives et objectives (F. Boillot, 1998, 2002). Ces méthodes reflètent la place centrale donnée aujourd'hui au visiteur dès la conception même du projet muséal. De fait, la scénographie du musée est de plus en plus centrée sur les préoccupations du public et sur les questions d'identité. (J. Davallon et Al., 1992).

Le projet de médiation se doit de révéler l'ensemble des interprétations à l'œuvre sur la biodiversité : des interprétations les plus immédiates aux plus codées, des plus indicielles aux plus symboliques. Par là, la biodiversité est une interprétation plurielle qui considère les interprétations des émetteurs les plus divers, celles des scientifiques, des institutions, des habitants mais aussi des artistes et des visiteurs.

\section{La biodiversité, une interprétation en constante transformation}

Cela posé, il convient aussi de remarquer que ces interprétations, une fois émises, ne sont pas fixes. Elles sont plus ou moins relayées, appuyées, rejetées, déformées selon leur efficacité symbolique, médiatique, politique. Comme le souligne D. Bougnoux (1995), ce que l'on suppose être le réel et la culture n'a jamais cessé d'être un artefact local que l'on s'efforce matériellement el laborieusement de généraliser en construisant certains réseaux. Ainsi opère la mise en culture de la science. Ici s'interroge la médiologie au sujet de l'efficacité symbolique d'une idée, de ce qui fait qu'elle réussit et se transforme. L'héritage culturel et naturaliste à transmettre par l'exposition apparaît pérenne (accumulé, construit, hérité) mais aussi évolutif.

J'insisterai sur le poids à donner à la dynamique qui anime la biodiversité, à son fonctionnement et à sa transformation. Je retiendrai aussi l'importance de la composante temporelle et notamment de l'ancrage de la thématique dans le passé, le présent et le futur.

Dès lors, il apparaît évident que, même issu des meilleures sources scientifiques, le matériau d'une exposition sur la biodiversité ne peut se réduire à un ensemble statique d'éléments naturels, culturels et artificiels ${ }^{8}$ qui ici conditionnerait la vie en général et la vie humaine en particulier. L'écologie nous fournit là un modèle opératoire. En effet, en écologie, la définition de la biodiversité s'appuie sur une approche intégrée des éléments (de la molécule, l'espèce, la population à l'écosystème) mais surtout, elle repose essentiellement sur la diversité et la qualité des processus fonctionnels du vivant. Et ces processus sont à considérer comme larges, complexes, évolutifs, incertains, méconnus et nécessaires à la vie d'un point de vue écologique, éthique, culturel, économique et social (F. Ramade, 2002).

De fait, la biodiversité doit être vue en ternies de qualité plus que de quantité et selon la perspective de la propriété des systèmes ou encore de leur fonctionnement.

21 En résumé, cette définition qualitative et ses composantes proposent ainsi un axe conceptuel majeur de la thématique biodiversité (cf. Figure I. A. point 1. et point 2.1.,la valeur écologique) à 
condition bien sûr, de l'insérer dans la dynamique identitaire territoriale et de l'ouvrir à une vue pluridisciplinaire et pluridimensionnelle et à une interprétation rassemblant tous les types et les profondeurs de regards potentiels, le noterai que, outre qu'il livre un axe structurant et contemporain de la médiation de la thématique, ce modèle rencontre particulièrement le modèle de la communication centré aussi sur la relation et sur sa qualité, sur les processus fonctionnels et sur leur dynamique systémique.

\section{La biodiversité, une notion structurée par l'éthique}

Si elle est avant tout une dynamique, la thématique "biodiversité » doit aussi rester manipulable. Il convient de situer les grandes lignes de force du contenu qui, ensemble, participent à approcher une définition idéale de la biodiversité. Cette définition exprime obligatoirement une tension. Car rappelons que chaque projet de médiation aura sa propre définition contextualisée, c'est-à-dire issue d'une négociation entre les acteurs en présence. La définition idéale devrait donc aussi constamment mettre en visibilité les types de positionnements possibles et avoir pour finalité de favoriser une mise en débat productive et prospective. Une telle plateforme pluridimensionnelle nous est fournie par la réflexion éthique directement liée à la tension vive qui existe actuellement entre écologie et socioéconomie. Je m'appuierai ici amplement sur le travail conséquent de $\mathrm{M}$. Chauvet et L. Olivier (1993) qui distinguent cinq valeurs individualisées et complémentaires associées aujourd'hui à la biodiversité. Ces valeurs en interaction sont la valeur écologique, la valeur d'usage, la valeur patrimoniale, la valeur d'option et la valeur d'existence (cf. Figure I. A., point 2.1.).

\section{La valeur écologique : la biodiversité biologique (cf. Figure I. A., point 2.1.)}

Il est ici essentiel de faire comprendre que la conservation et la gestion de la biodiversité ne revient pas à conserver statiquement toutes les espèces mais à tenter d'assurer les processus de renouvellement d'un grand nombre d'espèces et d'écosystèmes.

La priorité de médiation doit concerner ici l'appréhension des principales lois écologiques, l'approche intégrée des différents niveaux biologiques mais surtout l'appréhension de "la relation "sous toutes ses formes. ${ }^{9}$

Il convient d'insister sur la complexité et l'ampleur des processus fonctionnels et sur le fait que celles-ci posent des questions non résolues aux chercheurs et aux gestionnaires. Elles situent l'enjeu à venir des politiques de recherche et le caractère dynamique et évolutif que doit prendre un discours de conservation (cf. figures LA et I.B, points 3).

D'un point de vue didactique, l'appréhension de cette ampleur reposera sur une approche intégrée du vivant et sur les liens entre microsystème et macrosystème, entre échelle locale et échelle globale, entre passé, présent et futur. Elle mettra en relation complexité, échelles et incertitudes introduisant ainsi les fondements du principe de précaution et les enjeux des recherches menées notamment par le Parc national et ses partenaires.

À l'intérieur de ces processus fonctionnels, la biodiversité écologique est liée à la spéciation. Cela fait référence à la notion d'espèce, à la liste qualitative et quantitative d'espèces, aux notions liées aux mécanismes de spéciation voire d'extinction. Étant 
donnée la dominance de la perspective fonctionnelle de la thématique biodiversité, il convient de (M. Chauvet et L. Olivier, 1993) :

Valoriser l'espèce et l'écosystème par rapport à l'individu et dégager non pas une liste d'espèces mais des espèces « clés de voûtes » d'un point de vue écologique (c'est-à-dire des espèces dont dépendent un grand nombre d'espèces et l'écosystème même) et d'un point de vue culturel, esthétique et socioéconomique (par exemple, le parent sauvage des plantes cultivées...).

D'un point de vue didactique, il est important de mettre l'accent sur la différence entre les espècesclés et les espèces dont on parle parce qu'elles touchent l'affect et les effets de mode. Ces dernières ont plus leur raison d'être par stratégie didactique (accroche) que par fondement thématique (objectifs, contenus). La dérive d'une médiation basée uniquement sur des espèces-phares fait perdre de vue le fonctionnement même de l'environnement et la primauté de "la relation" par rapport a "l'individu ». Elle aboutit à une vue simpliste et peu opérationnelle de l'écogestion.

D'ouvrir rapidement à une réflexion large dépassant les espèces, centrée sur la relation et englobant tous les niveaux biologiques et toutes les échelles d'espace et de temps.

31 Outre l'intérêt des contenus écologiques précédemment développés, la mise en visibilité des systèmes et de leur dynamique permet aussi de s'éloigner d'une représentation "nature-somme d'objets » comme d'échapper aux obstacles didactiques générés par l'expérience concrète. Par exemple, l'apparente densité localisée d'une espèce rare rend le message de protection incompréhensible. Cela implique de replacer cette densité relative dans une vue plus large établissant par exemple, les notions de population minimale et d'ampleur écologique.

De lier ces notions aux autres valeurs ci-dessous (cf. Figure I.B, points 2.2 à 2.5) et à la quatrième perspective de la biodiversité, c'est-à-dire les politiques et les actions de conservation, d'écogestion et de développement durable (cf. Figures LA et I.B, points 3.1 et 3.2) qui.mimeront une partie du débat muséal.

\section{La valeur d'usage : la biodiversité socioéconomique (cf. Figure I.B, point 2.2.)}

Elle réfère à l'utilisation de la nature, sous toutes ses formes, par l'homme. Cette valeur relève l'enjeu socioéconomique de la diversité des gènes, des espèces et des milieux susceptibles d'être utilisés par l'homme : les milieux « dits naturels » ou très transformés, les animaux «sauvages » et/ou domestiqués, les plantes "sauvages » et/ou cultivées, c'est-à-dire tous les milieux, animaux et plantes simplement utilisés, de façon directe ou indirecte ${ }^{10}$, ou éventuellement modifiés et/ou introduits par l'homme. Les finalités de ces usages peuvent être économiques (productions agricoles, sylvicoles, ornementales..), médicales (aromatiques, médicinales ${ }^{11}$ ), récréatives (cueillette, pêche, chasse, loisirs...), culturelles (espèces ou habitats symboliques et/ou identitaires de communautés et d'histoires humaines; création artistique), urbanistiques (matériaux d'architecture; énergie; amélioration bioclimatique de l'habitat; esthétique..), sécuritaires (végétalisation des berges, marais de lagunage, végétalisation pare-feu) voire scientifiques (la science considérée ici comme un lieu de production de connaissances) (M. Chauvet et L. Olivier, 1993). 

habitats et par une forte érosion génétique (cf. Figure I.B, points 2.6 et 2.7.). Celle-ci est notamment due à l'harmonisation des marchés et au développement d'une agriculture industrielle basée sur la production d'une gamme réduite d'espèces phares peu diversifiées. Cette érosion génétique se traduit par la disparition de nombreuses espèces domestiques ou cultivées voire simplement associées à ces espèces, c'est-à-dire par la perte de différentes propositions génétiques faites au fil du temps par la biodiversité en réponse aux conditions de vie micro-locales. L'érosion génétique est, entre autres, liée à la destruction des milieux naturels ou transformés, au profit de monocultures uniformisant le territoire. Ceci induit aussi la perte d'espèces «sauvages» qui représentent les «parents sauvages des espèces cultivées » et, à ce titre, constituent une banque de gènes susceptibles d'être utilisée lors de l'apparition de nouveaux besoins. Outre les pertes spécifiques et intraspécifiques, l'érosion génétique suppose une perte de potentiel adaptatif évident face à de futurs changements écologiques (par exemple, changement climatique ou apparition d'espèces dominantes par dispersion écologique ou transfert de gènes, etc.), à de futurs changements culturaux (apparition de nouvelles maladies ${ }^{12}$; résistance aux traitements chimiques, etc.) et économiques (nouveaux marchés ${ }^{13}$, nouveaux besoins des consommateurs, etc.) (M. Chauvet et L. Olivier, 1993).

édiation de la valeur d'usage met particulièrement eu visibilité les relations homme-nature, l'imbrication entre nature "naturelle » et " artificialisée », et par là, l'impact obligatoire positif et/ ou négatif que l'homme a sur son environnement. Cette valeur permet dont de questionner la responsabilité individuelle et collective vis-à-vis de la biodiversité. Elle ouvre aussi directement à la socioéconomie et permet une réflexion sur un développement dit durable. Enfin, la valeur d'usage a un sens plus immédiat et plus concret que les autres valeurs notamment que la valeur écologique qui nécessite l'intégration de concepts abstraits et spécialisés. La valeur d'usage favorise donc la rencontre du grand public avec l'ensemble de la thématique. Néanmoins, il conviendra ici d'encadrer une habituelle représentation militariste de la nature qui, si elle est importante, a tendance à faire obstacle à l'appréhension des autres valeurs.

ailleurs, la valeur d'usage renvoie aussi à un usage symbolique et culturel de l'espace et de ses composantes. Les transformations génétiques, spécifiques ou écosystémiques opérées par plusieurs générations humaines et ce, depuis le néolithique, sont le fruit et le témoin des mythes, des religions, des économies, des savoir-faire et des cultures des acteurs de ces transformations. ${ }^{14}$

La biodiversité est donc ici un véritable marqueur culturel sous-tendant des savoir-faire, des traditions et des cultures. Elle constitue aussi un témoin de l'histoire de l'origine et de l'amélioration des espèces et des habitais. Elle est, de plus, un réservoir potentiel des connaissances empiriques sur la variabilité et l'adaptation des espèces, un réservoir qui intéresse aujourd'hui la recherche écologique et agronomique.

38 Ainsi la valeur d'usage identifie particulièrement la valeur patrimoniale et la valeur d'option qu'il importe de relever en tant que telles.

\section{La valeur patrimoniale : la biodiversité, marqueur culturel et écologique (cf. Figure I.B, point 2.3.)}

La valeur patrimoniale situe le gène, l'espèce et l'habitat naturel ou humanisé comme la résultante d'un processus unique mené sur plusieurs millions d'années avec ou sans 
l'homme. Ils sont témoins particuliers d'une nature, d'une culture, d'une esthétique, d'une pratique et d'un passé, d'un présent voire d'un avenir. Ils sont marqueurs culturels ou/et écologiques. Ils contribuent à la constitution d'un patrimoine mondial au même titre qu'un monument historique ou qu'une œuvre d'art. Ils constituent une mémoire des processus et ainsi un réservoir potentiel de connaissances (M. Chauvet et L. Olivier, 1993).

\section{La valeur d'option : la biodiversité, réserve d'adaptabilité (cf. Figure I.B, point 2.4.)}

La pensée associée à la valeur d'option prône un maintien de la biodiversité des populations et des habitats, une biodiversité vue comme "une réserve d'adaptabilité » aux futurs changements écologiques et socioéconomiques. Elle s'appuie sur notre méconnaissance des phénomènes génétiques et écologiques. Elle s'interroge sur les rôles écologiques et socioéconomiques potentiels à venir des nombreuses espèces dont la fonction des gènes est inconnue ou des espèces non encore identifiées. Elle s'inquiète sur le niveau de menaces engendrées par l'actuelle érosion génétique et sur les capacités de réponses potentielles et invisibles des habitats et de leurs espèces naturelles et domestiques (cf. Figure I.B, points 2.6 et 2.7.). Mais aussi, elle pose la question des menaces locales ou globales à venir et des changements probables des écosystèmes; ou encore celle des impacts des mutations à venir ou des transferts horizontaux de gènes, notamment dans le cas des plantes transgéniques. Enfin, elle conduit à souligner notre méconnaissance de nos futurs besoins économiques, alimentaires, médicaux, culturels, touristiques, etc. et donc de celles de nos attentes vis-à-vis de la biodiversité (M. Chauvet et L. Olivier, 1993). Dans la mesure où il est impossible de dire quels gènes, quelles espèces ou quels habitats seront les plus aptes à s'adapter aux changements climatiques, écologiques et socioéconomiques, la valeur d'option invite à protéger la biodiversité et ce, le plus largement possible.

\section{La valeur d'existence : la vie pour la vie (cf. Figure I.B, point 2.5.)}

Elle fait directement appel à une réflexion éthique car elle propose de respecter non seulement la vie pour l'homme mais la vie pour la vie. Il s'agit de reconsidérer le rapport homme-nature en équilibrant la vision utilitariste de la nature par un sentiment d'appartenance en tant qu'espèce vivante dans la nature. Concrètement, cela correspond à respecter pour eux-mêmes les habitats, les espèces et les éléments biotiques et abiotiques (abiotiques au sens des éléments sans vie comme les roches, par exemple), mêmes ceux qui apparaissent à priori inutiles (M. Chauvet et L. Olivier, 1993). Cette valeur élargit considérablement le principe de responsabilité et les débats sur l'éthique du vivant. Elle est importante face à l'utilitarisme et le positivisme ambiants.

\section{La biodiversité comme lieu de débat}

L'élaboration d'une grille conceptuelle sur la biodiversité repose ici sur un parti pris. Il pose comme préalable une définition de la biodiversité obligatoirement liée à son contexte et par la, une mise en oeuvre d'une négociation opérée dans chaque projet de médiation et de territoire. Cette négociation doit aussi s'inscrire dans plusieurs niveaux interreliés, du local au global, et intégrer passé, présent et futur. Une telle élaboration a 
pour finalité une mise en débat et en perspective de la biodiversité. Il s'agit de rejoindre la dernière fonction plus impliquante du musée qui le conduit à affirmer sa relation avec le territoire et ses acteurs, voire à jouer un rôle d'animateur et de médiateur des problématiques locales (J. Davallon et AL, 1992). Si la dynamique de la biodiversité se structure sur la base de cinq valeurs imbriquées, leur mise en dialogue est donc essentielle au projet de médiation. Ce dialogue s'inscrit dans un climat positif (notre appartenance au vivant dans toutes ses dimensions) mais aussi fortement négatif (le renvoi aux menaces qui pèsent sur la biodiversité). En effet, la prégnance de la dimension sécuritaire dans notre société n'épargne pas le domaine de l'environnement (J. Theys, 1993). C'est pourquoi j'ai listé les principales menaces et leurs conséquences le plus fréquemment relevées (Figure I.B, points 2.6 et 2.7). Néanmoins, il importe de rester dans une éthique positive de médiation (U. Lecourt, 1993), en pensant un projet qui porte en lui non pas un catastrophisme lié à la résolution de problèmes mais un souci d'optimisation des qualités de notre environnement.

Le projet de médiation a donc pour visée principale la valorisation et la mise en débat d'une conservation et d'une écogestion dynamiques et plus largement d'un développement dit durable, c'est-à-dire un développement qui répond aux besoins $\mathrm{du}$ présent sans compromettre la capacité des générations futures de répondre aux leurs (Commission mondiale sur l'environnement et le développement, 1988).

Les Figures LA et I.B. (points 3.1 et 3.2) reprennent les éléments du débat, c'est-à-dire les actions à envisager du point de vue de l'écogestion stricte, les enjeux des recherches et des métiers du Parc national et de ses partenaires et les types de protection envisageables. Ce débat réunit aussi bien des experts, des gestionnaires, des décideurs que de simples citoyens. Il permet aussi une confrontation active entre de nombreuses visions de l'environnement qu'elles soient anthropocentriques (l'homme domine une « nature somme de ressources", l'homme est surtout culturel), biocentriques (la nature domine l'homme, l'homme est surtout biologique) ou technocentriques (la nature et l'homme sont interdépendants mais l'environnement est vu en termes de risques et de menaces, l'homme est surtout l'homme du souci) (J. Theys, 1993). De fait, pour reprendre les termes de L. Olivier et M. Chauvet (1993), la diversité des points de vue sur la diversité est le meilleur garant du maintien de cette dernière. De fait, le projet de médiation s'adresse à tous les visiteurs potentiels (personnels du Parc, institutions partenaires, décideurs, habitants, touristes, etc.). Et il appelle à la responsabilisation individuelle et collective voire à la participation aux politiques territoriales afin de se situer idéalement comme une aide au changement.

\section{Le projet de médiation (cf. Figure II)}

La clarification conceptuelle de la biodiversité établie, le concepteur du projet de médiation peut opérer une sélection thématique. Cette sélection va dépendre de nombreux guides bien connus en muséologie (P. Rasse, 1997). Mon intention n'est pas de les développer ici, mais je rappellerai simplement, dans la figure IL, les grands axes du projet culturel et scientifique de l'exposition. La clarification du concept de la biodiversité est placée en position centrale et préalable. On y retrouve les lignes de force précédemment développées. La dynamique, le fonctionnel et la relation priment sur l'objet. Les cinq valeurs structurent le thème. Le débat oriente le projet. Puis, l'opération de sélection thématique et stratégique est guidée par une série de questions. Il s'agit de se 
demander comment le mieux servir le projet de communication des commanditaires et de leurs partenaires, c'est-à-dire ici les finalités muséales liées aux quatre missions du Parc national? Comment élaborer un pro)et original, révélateur d'un territoire particulier? Comment mettre en visibilité et en synergie l'identité du site et des sites de proximité, l'identité de la vallée et celle du Parc national du Mercantour? Comment définir et délimiter le média in et ex-situ et l'insérer dans le concert médiatique des territoires ? Comment respecter les diversités des publics et des moments de visite ? Comment parler à la fois aux habitants et aux touristes, aux institutions et aux décideurs? Comment juxtaposer les objectifs éducatifs et établir une progression du niveau opératoire des messages adaptée aux cibles? Comment articuler les quatre temps forts d'une médiation environnementale: l'immersion, la contextualisation, l'éducation et l'appel à participation? Autant de questions qui constituent un outil d'aide à la concertation et à la conception.

\section{Conclusion}

L'enjeu de mes recherches sur les Parcs nationaux est de comprendre, aménager et mettre en scène l'espace sans en dégrader ni l'essence ni le sens tout en questionnant un modèle d'investigation actuel liant écologique et symbolique, images universelles et singularité des natures et des cultures et favorisant dialogue et participation élargie des acteurs en faveur d'un développement durable. Ce projet par trop ambitieux nécessite de changer fréquemment d'échelle, de lieu et de niveau d'interrogation. Longtemps concentrée sur la dynamique générale du projet de médiation d'un vaste «territoire Parc national ", j'ai eu à cœur ici de revenir à un moment limité du projet, délimité spatialement et thématiquement. Si j'ai rappelé la nécessité de replacer, par principe, chaque média dans le concert médiatique du territoire et dans son cadre institutionnel, politique et communicationnel, mon propos a été surtout de situer la clarification conceptuelle de la thématique "biodiversité ». La définition de la biodiversité s'affirme comme le résultat d'une négociation permanente. Pluridisciplinaire, pluridimensionnelle, elle rassemble idéalement tous les interprétants possibles (du scientifique à l'artiste, de l'habitant au visiteur, etc.) et tous les niveaux d'interprétation, des plus indiciels aux plus codés. La biodiversité se dessine aussi comme un concept vivant, dynamique, fonctionnel et animé par cinq valeurs : la valeur écologique, la valeur d'usage, la valeur patrimoniale, les valeurs d'option et d'existence. En constante transformation et discussion, elle rassemble ou sépare les protagonistes du média et, en tout cas, permet d'animer la thématique dans et hors l'exposition, et ce faisant un territoire, son identité et son projet.

BIBLIOGRAPHIE

BEGUIN F., Le paysage, Editions Flammarion, Paris, 1998.

BERQUE A., Médiance de milieux en paysages, GIP-Reclus, Montpellier, 1990. 
BOILLOT-GRENON F., « Effet de loupe sur la question de l'objet : le cas d'un espace muséal de 250 000 hectares ", in : Des expositions scientifiques à l'action culturelle, des collections pour quoi faire? Colloque international de muséologie, Muséum national d'histoire naturelle, 6-7 juillet 1998, Paris, 1998.

BOILLOT-GRENON F., «Clarifier les représentations des partenaires de l'éducation relative à l'environnement », in : L'école et ses partenaires scientifiques, ASTER, n 29, INRP, Paris, 1999.

BOILLOT-GRENON F., " Le visiteur, acteur de la médiation des parcs nationaux », in : Espaces et territoires : du public à l'habitant, Colloque du 6-7 juin 2002, à paraître, Université de Lille III, Roubaix, 2002.

BOILLOT-GRENON F., (sous presse), « La transmission du patrimoine comme projet de territoire », dans : Olfaction et patrimoine : quelle transmission? Actes du colloque, (sous la direction de F. Boillot-Grenon et M.-C Grasse), Musée International de la Parfumerie de Grasse, Edisud, Aix-enProvence, 2004.

BOUGNOUX D., Introduction aux sciences de l'information et de la communication, Editions La découverte, Paris, 1998.

BOUGNOUX D., La communication contre l'information, Hachette, Paris, 1995.

CHAUVET M., OLIVIER L., La biodiversité, enjeu planétaire, Editions du Sang de la Terre, Paris, 1993. CORAJOUD M., " Le paysage, c'est l'endroit où le ciel et la terre se touchent », dans : Mort d'un paysage f Philosophie et esthétique du paysage, (sous la direction de F. Dagognet), Editions Champs Vallon, Seyssel, 1982.

DAGOGNET F., (sous la direction de), Mort d'un paysage? Philosophie et esthétique du paysage, Editions Champs Vallon, Seyssel, 1982.

DAVALLON J., GRANDMONT G., Schiele B., L'environnement entre au musée, PUL/MC, Lyon-Québec, 1992.

DEBRAY R., Transmettre, Editions Odile Jacob, Paris, 1997.

DE CERTEAU M., La culture au pluriel, Le Seuil, Paris, 1993.

LECOURT D., Contre la peur, suivi de critiques de l'appel d'Heidelherg, Hachette, Paris, 1993.

OCDE, Etat de l'environnement, OCDE, Paris, 1991.

PEIRCE G, Ecrits sur le signe (traduit par G. Deledalle), Le Seuil, Paris, 1978.

RASSE P., Techniques et cultures au musée, PUL, Lyon, 1997.

RAMADE F., Eléments d'écologie fondamentale, Edisciences Editions, Paris, 2002.

RIVIERE G.H., Musées et société, à travers le temps et l'espace, la muséologie selon G.H. Rivière, Bordas Dunod, Paris, 1989.

SAUVE L., Pour une éducation relative à l'environnement -Eléments de design pédagogique, Editions Guérin / Montréal, Editions Eska, Paris, 1994.

THEYS J., L'environnement, à la recherche d'une définition, Note de méthode $n$ 1, IFEN, Orléans, 1993.

VIEL A., Quand souffle l'esprit des lieux : nature et culture au diapason de la pérennité, Patrimoine culturel, patrimoine naturel, Colloque, Ecole nationale du patrimoine. La documentation française, Paris, 1994. 


\section{NOTES}

1. Cette maison est elle-même contiguë.au Musée des Merveilles qui expose l'occupation humaine de la vallée des Merveilles, un site célèbre pour ses gravures rupestres.

2. Rappelons que G.H. Rivière (1989), définit un musée comme un laboratoire, un conservatoire, une école, un lieu de participation de notre temps. Avec ou sans toit.

3. Les crises, notamment identitaires, sont fréquentes dans ces zones de haute montagne marquées par la déprise agricole.

4. Il s'agit d'un outil d'aide à la décision appelé schéma d'interprétation développé en 1998 dans le Parc des Ecrins et en 2001 d.ins le Parc national de Port-Cros.

5. Lors de précédents travaux, j'ai ainsi appelé à une conciliation entre quatre producteurs de sens, entre quatre paysages émergeant a partir d'un même espace : le paysage inventé, mouvant, contemporain du visiteur; le paysage de l'habitant tissé de masques et de marqueurs culturels communautaires ; le paysage de l'institution Parc national décrypté a travers le prisme des deux missions dominantes que sont la conservation et la science; le paysage du médiateur, a priori le plus équilibré mais, souvent déformé par une vision pédagogique et moralisatrice (F. Boillot, 1998).

6. Classiquement lieu de conservation et de recherche, le musée s'affirme aujourd'hui comme lieu d'apprentissage et de changement social (J. Davallon, 1992).

7. Notons aussi qu'outre la vertu intrinsèque et éducative d'une définition globale, la dimension écologique et sécuritaire a l'avantage de respecter l'identité de l'émetteur l'arc national et la dimension socioéconomique et culturelle permet de miens rencontrer une grande partie des visiteurs mats aussi des habitants valléens, ce qui augure d'une relation facilitée.

8. D'autres arguments jouent contre ce choix. Il réfère à la muséologie d'objets qui est un modèle largement dépassé au profit d'une muséologie de la connaissance ou de celle de l'implication (J. Davallon, 1992). Par ailleurs, il est susceptible de génerer une représentation où la nature est lue comme un vaste supermarché, avec un étalage d objets. Ce qui renforce des attitudes de consommation voire de déprédation, incompatibles avec les objectifs d'une éducation relative à l'environnement.

9. Cette vision systémique est difficile à appréhender pour des publics néophytes. Il convient d'encadrer la compréhension de plusieurs notions essentielles mais délicates, par exemple les notions de cycle, de système, de rétroaction ; ou l'idée d'une nature avançant par essai el erreur ; ou encore le fait que les extinctions actuelles posent problème au vivant même si les extinctions sont des phénomènes normaux et même si les perturbations ont toujours existé. Notons que, selon M. Chauvet cl O. Olivier (1993), actuellement 140 mammifères sont en danger et 2 lois plus d'espèces ont disparu depuis 400 ans par rapport au rythme habituel. El le rythme d'extinction est évalué à un mammifère tous les 400 ans el à un oiseau tous les 200 ans.

10. Voir, par exemple, le rôle indirect des insectes sauvages sur la pollinisation des arbres fruitiers.

11. Plus de la moitié des médicaments dans le monde provient des plantes et beaucoup de modèles biologiques servent à la fabrication des modèles chimiques (exemple, l'aspirine) (M. Chauvet et O. Olivier, 1993).

12. Cf. les ravages du mildiou en Irlande ou du phylloxera en France.

13. Cf. par exemple, le nouveau marche actuel du riz sauvage ou l'engouement pour des ornementales diversifiées et proches des espèces sauvages (M. Chauve et O. Olivier, 1993).

14. Voir par exemple, la civilisation euro-méditerranéenne liée au blé, à l'olivier et à la vigne ; ou encore, la biodiversité des raisins plus développée pour le raisin de table dans les pays musulmans et pour le raisin à vin clans les pays judéo-chrétiens (M. Chauvet et O. Olivier, 1993). 


\section{RÉSUMÉS}

Les identités locales disparaissent ou se banalisent comme les propositions muséales associées. De fait, il importe de relever l'ambition du projet de médiation des territoires. Une exposition sur la biodiversité du Parc national du Mercantour donne ici l'occasion de revaloriser la clarification conceptuelle du contenu de la médiation et par là, celle du thème actuel de la biodiversité. Celleci se dessine comme un concept vivant, fonctionnel et dynamisé par cinq valeurs éthiques. Mais elle est surtout le résultat d'une négociation contextuelle permanente qui rassemble ou sépare les protagonistes du média et qui permet de valoriser et d'animer, dans et hors l'exposition, un territoire, son identité et son projet.

The local identities vanished or turn in common places as the associaled muséum displays. Then. il is important lo set up the scope of comniunication's project of the areas. An exhibition on the biodiversity of the Mercantour National Park is the opportunity to clarify the contents of the communications project and also of the fashionable theme of the biodiversity. Biodiversity appears as a living, dynamic and functional concept based on five ethical values. But il is moreover the result ofa permanent local negociation wich put logether or separate the protagoniste of the project and wich allow, within and outside of the exhibition, the valorisation and the animation of the area, ofits identity and project.

\section{AUTEUR}

\section{FRANCINE BOILLOT-GREN}

Francine Boillot-Grenonest à la croisée des chemins. Chemins des espaces protégés, chemins des médiations culturelles, chemins des disciplines pour parcourir la complexité d'un endroit précis du territoire. 